\title{
Discovery of Enhanced Germanium Abundances in Planetary Nebulae with the Far Ultraviolet Spectroscopic Explorer
}

\author{
N. C. Sterling ${ }^{1}$, Harriet L. Dinerstein ${ }^{1}$, and Charles W. Bowers ${ }^{2}$
}

\begin{abstract}
We report the discovery of Ge III $\lambda 1088.46$ in the planetary nebulae (PNe) SwSt 1, BD $+30^{\circ} 3639$, NGC 3132, and IC 4593, observed with the Far Ultraviolet Spectroscopic Explorer ${ }^{1}$. This is the first astronomical detection of this line and the first measurement of $\mathrm{Ge}(Z=32)$ in $\mathrm{PNe}$. We estimate Ge abundances using $\mathrm{S}$ and $\mathrm{Fe}$ as reference elements, for a range of assumptions about gas-phase depletions. The results indicate that Ge, which is synthesized in the initial steps of the $s$-process and therefore can be self-enriched in $\mathrm{PNe}$, is enhanced by factors of $\geq 3-10$. The strongest evidence for enrichment is seen for PNe with Wolf-Rayet central stars, which are likely to contain heavily processed material.
\end{abstract}

Subject headings: line: identification-planetary nebulae: general-planetary nebulae: individual (BD+30³639, SwSt 1, IC 4593, NGC 3132) — stars: AGB and post-AGBnucleosynthesis, abundances - ultraviolet: ISM

\section{INTRODUCTION}

Asymptotic giant branch (AGB) stars, the progenitors of planetary nebulae (PNe), are believed to be the primary synthesis site for heavy element isotopes produced by the slow neutron-capture or $s$-process (Käppeler et al. 1989; Käppeler 1999). Since enhanced surface abundances of $s$-process products are observed in evolved red giants (e.g. Smith \& Lambert 1990), it is reasonable to expect such enrichments in PNe as well. Péquignot \& Baluteau (1994) reported optical emission lines indicating overabundances for a large number of $n$-capture elements in the PN NGC 7027, and Dinerstein (2001) identified two near-infrared lines commonly seen in PNe as fine-structure transitions of the light $n$-capture elements $\mathrm{Kr}$ and $\mathrm{Se}(Z=34,36)$. Germanium $(Z=32)$ is more abundant than Kr and Se in the solar system, and, like these elements, can be synthesized in AGB stars of roughly solar metallicity (Busso et al. 1999; 2001).

\footnotetext{
${ }^{1}$ Astronomy Department, University of Texas, Austin, TX 78712-1083

${ }^{2}$ Laboratory for Astronomy and Solar Physics, Code 681, NASA Goddard Space Flight Center, Greenbelt, MD 20771

${ }^{1}$ Based on observations made with the NASA-CNES-CSA Far Ultraviolet Spectroscopic Explorer. FUSE is operated for NASA by Johns Hopkins University under NASA contract NAS5-3298.
} 
In this Letter, we report the discovery of the strong resonance line Ge III $\lambda 1088.46$ in four PNe observed with FUSE, and present evidence that Ge has been self-enriched in their precursor stars.

\section{OBSERVATIONS AND DATA REDUCTION}

FUSE (Moos et al. 2000; Sahnow et al. 2000) observations of BD+30 3639 and SwSt 1 were obtained for General Investigator (GI) programs A085 and B069 (P.I. Dinerstein) in timetag mode using the LWRS aperture $\left(300^{\prime \prime} 0 \times 300^{\prime \prime} 0\right)$. NGC 3132 was observed for program P133 (P.I. Bianchi), also with the LWRS in time-tag mode, while IC 4593 was observed with the HIRS aperture $\left(1{ }^{\prime \prime} .25 \times 20^{\prime \prime} 0\right)$ in histogram mode for GI program B032 (P.I. Gruendl). The FUSE data and observational information may be accessed from the MAST archive at the Space Telescope Science Institute under the indicated GI programs.

The individual exposures were calibrated with CALFUSE v2.0.5, and all exposures for a given object were cross-correlated and co-added to increase the signal-to-noise ratio $\mathrm{S} / \mathrm{N}$. We based our subsequent analysis on the detector segments with the highest $\mathrm{S} / \mathrm{N}$ in the salient wavelength regions: LiF $2 \mathrm{~A}$ for Ge III $\lambda 1088.46$ and the Fe III lines at $\lambda \lambda 1122-1132$, and LiF 1A for S III $\lambda \lambda 1012-1021$. In cases of overlapping coverage, the results were reasonably consistent in both detector segments. The spectra were rebinned by 3 pixels, increasing the $\mathrm{S} / \mathrm{N}$ near $1088 \AA$ to $15-25$ per rebinned sample, and broadening the sampling interval from $\sim 7 \mathrm{~m} \AA$ to $\sim 20 \mathrm{~m} \AA$.

The continua were normalized and flattened by fitting low-order Legendre polynomials to the continuum shape and dividing them out. We then fit the lines with single or multi-component Gaussian profiles after Sembach et al. (1993) convolved with a single Gaussian instrumental profile of FWHM $20 \mathrm{~km} \mathrm{~s}^{-1}(R=15,000)$. The normalized profiles are shown in Figure 1, with the fits for nebular features overplotted as thick solid lines. Other lines which were modeled to constrain the fit of the nebular features are shown as dashed lines. The dotted vertical lines indicate nebular absorption velocities from Na I (Dinerstein et al. 1995) or from other lines in the FUSE spectrum.

In Table 1 we summarize the values of central velocity $\langle v\rangle$ and equivalent width $\mathrm{W}_{\lambda}$ from the line fits. The Doppler widths $b$ are poorly determined because they are small compared to the FUSE instrumental resolution element; typical fitted values were $b \sim 10 \mathrm{~km} \mathrm{~s}^{-1}$. The errors are $1 \sigma$ values based on uncertainties in continuum placement and statistical errors in the fitting process; upper limits are $3 \sigma$. Wavelengths and data for atomic transitions are from Morton (1991, 2000). Data on $\mathrm{H}_{2}$ transitions were obtained from $\mathrm{S}$. McCandliss (private communication based on Abgrall et al. 1993 a,b; also see http://www.pha.jhu.edu/ stephan/H2ools/h1h2data/). 


\section{RESULTS}

\subsection{Identification of $\lambda \mathbf{1 0 8 8 . 4 6}$ as Ge III}

We identify the feature centered at rest wavelength $\lambda 1088.46$ as an intrinsically strong Ge III line (oscillator strength $f=1.84$, Morton 2000). Since $\mathrm{Ge}^{++}$is located in the H II region, it should have the same velocity as other ionized species. SwSt 1 and IC 4593 show good agreement (Table 1), while the Ge III lines appear at slightly more negative velocities in BD+30 3639 and NGC 3132. However, these discrepancies are probably not significant given the resolution of FUSE.

Since the ionization potentials (IPs) of Ge II and Ge III are $15.9 \mathrm{eV}$ and $34.2 \mathrm{eV}$ respectively, we expect Ge III to contain a large fraction of the gas-phase Ge in PNe with relatively cool central stars, where O II comprises a significant fraction of O. (In comparison, $\operatorname{IP}(\mathrm{O}$ I) $=13.6 \mathrm{eV}$ and $\mathrm{IP}(\mathrm{O} \mathrm{II})=35.1 \mathrm{eV}$.) SwSt 1 and $\mathrm{BD}+30^{\circ} 3639$ have cool $(T \sim 35,000 \mathrm{~K})$, late Wolf-Rayet $([\mathrm{WR}])$ type central stars and relatively high $\left(\mathrm{O}^{+}\right) /\left(\mathrm{O}^{++}\right)$. IC 4593 also has a fairly cool central star (Kaler \& Jacoby 1991). The central star of NGC 3132 is a visual binary consisting of an A2 V star plus a hotter, low-luminosity companion (Méndez 1978). We set upper limits on $N\left(\mathrm{Ge}^{+3}\right)$ based on non-detections of Ge IV $\lambda 1229.84(f=0.25)$ in STIS spectra of SwSt 1 (De Marco et al. $2002)$ and $\mathrm{BD}+30^{\circ} 3639$, and on $N\left(\mathrm{Ge}^{+}\right)$from $\lambda 1237.06(f=1.23)$ for SwSt 1 and $\mathrm{BD}+30^{\circ} 3639$, and $\lambda 1098.71(f=0.55)$ for IC 4593 and NGC 3132 .

We found no plausible alternate identifications for the feature near $\lambda 1088.46$. The nearest lines are $\mathrm{Cl}$ I $\lambda 1088.05$, with a wavelength offset corresponding to $-111 \mathrm{~km} \mathrm{~s}^{-1}$ relative to the rest wavelength of Ge III, and $\mathrm{H}_{2} \mathrm{P}(4) 2-0 \lambda 1088.79$, at $+91 \mathrm{~km} \mathrm{~s}^{-1}$, neither of which interfere with Ge III in these objects. This wavelength region also contains several lines from $v \geq 1$ levels of $\mathrm{H}_{2}$, e.g. Lyman 2-3 $R(0), 2-3 R(1), 0-1 R(11)$, and 9-1 $R(11)$. Of our four targets, only BD $+30^{\circ} 3639$ and possibly NGC 3132 contain observable amounts of vibrationally excited $\mathrm{H}_{2}$. However, unblended lines from these levels with similar $f$-values have $\mathrm{W}_{\lambda} \leq 15 \mathrm{~m} \AA$ in $\mathrm{BD}+30^{\circ} 3639$ (Dinerstein et al., in preparation), and SwSt 1, with the strongest $\lambda 1088.46$ feature, does not show nebular $\mathrm{H}_{2}$ absorption at all. We therefore conclude that the observed features are primarily due to Ge III.

We also searched the archival FUSE spectra of 23 other PNe, but found no other detections of Ge III. However, various factors could be responsible for this, such as a weak UV continuum, central star hot enough to ionize most of the Ge beyond $\mathrm{Ge}^{++}$, or lack of sufficiently large column of ionized gas along the line of sight. Many PNe have asymmetrical structures, and because these measurements require a bright FUV continuum there is a selection bias in favor of nebulae for which the central star is seen along a low-extinction, low column-density sight line.

\subsection{Determination of Gas-Phase Column Densities}

The column densities listed in Table 1 were computed from the measured $\mathrm{W}_{\lambda}$ values, assuming that the lines are on the linear part of the curve of growth. In fact, some of the lines are marginally 
saturated. If the line widths were purely thermal $\left(b=1.5 \mathrm{~km} \mathrm{~s}^{-1}\right.$ for Ge, $2.3 \mathrm{~km} \mathrm{~s}^{-1}$ for $\left.\mathrm{S}\right)$, the line center optical depths would be $\tau_{0} \sim 5$. However, the fitted $b$ values are $\sim 10 \mathrm{~km} \mathrm{~s}^{-1}$, probably due to turbulence or shear motions, so that $\tau_{0}<1$ except for lines from the ground levels of the more abundant ions. In those cases we use lines from excited fine-structure levels, which are less saturated, to infer populations in the ground level.

The quantity of most interest is the Ge abundance with respect to an element which is unaffected by internal nucleosynthesis. Whenever possible, we use $\mathrm{S}$ as the reference element since it is not depleted into dust (Savage \& Sembach 1996), and assume that $(\mathrm{Ge} / \mathrm{S}) \sim\left(\mathrm{Ge}^{++}\right) /\left(\mathrm{S}^{+}+\mathrm{S}^{++}\right)$, as S II and S III together span a similar ionization range as Ge III (15.9-34.2 eV for Ge III; 10.4-23.3 $\mathrm{eV}$ for $\mathrm{S}$ II and $23.3-34.8 \mathrm{eV}$ for S III). We take Fe III (IP range 16.2-30.7 eV) as the reference species for NGC 3132 since we did not detect S III (see below).

Whereas Ge III has a $4 \mathrm{~s}^{2}$ ground configuration and therefore only one low-lying energy level, S III and Fe III possess several fine-structure levels which may contain significant populations. Ideally one should measure absorption lines from each level, but this is not always feasible. For S III, lines from both excited levels could be measured in all but NGC 3132. We used the web-based multi-level atom program of Shaw \& Dufour (1995; http://stsdas.stsci.edu/nebular) to predict the population ratios among the three fine-structure levels at the temperature and density of the ionized gas, and compared these with our derived column densities. If the measured population ratio of the excited levels agreed with the multi-level calculation, we use the latter to obtain the ground level population and total ionic column density.

SwSt 1 is the only object for which S II and transitions from all three levels of the ground ${ }^{3} \mathrm{P}$ term of S III are observable. The lines from the excited levels, S III* $\lambda 1015.78$ and S III** $\lambda 1021.33$, are free of blending, but the corresponding transition from the ground level at $\lambda 1012.50$ is potentially saturated and contaminated by several strong resonance $(v=0) \mathrm{H}_{2}$ lines. The value derived from this line is about $40 \%$ lower than the ground-level population inferred from the excited levels, which we attribute to saturation. We used S II $\lambda 1250.58$, a low- $f$ line in the STIS band, to derive $N(\mathrm{~S}$ II).

For $\mathrm{BD}+30^{\circ} 3639$, S III $\lambda 1012.50$ is not observable due to strong $\mathrm{H}_{2}$ absorptions from the nebula and foreground ISM, so $N(\mathrm{~S}$ III) in Table 1 is from S III $\lambda 1190.21$. However, since this line is saturated, we computed the ground-state population from the excited levels as described above, yielding a value about 2.6 times higher than measured. All of the S II lines are saturated, so we adopt S II/S III = 0.79 from Pwa et al. (1986) to infer $N(\mathrm{~S} \mathrm{II})$. We used a similar approach to determine ground state and total S III column densities for IC 4593, and obtained N(S II) by assuming the S ionic ratio from optical emission lines (Bohigas \& Olguín 1996).

Since S is not detected in NGC 3132, we were forced to use the less satisfactory species Fe III. The ${ }^{5} \mathrm{D}$ ground term of Fe III has several fine-structure levels, although the populations of the higher levels are likely to be small due to their low statistical weights. We marginally detect $\lambda 1124.88$, the strongest $(f=0.052)$ line from the first excited level, ${ }^{5} \mathrm{D}_{3}$. The surprising strength of $\lambda 1130.40$ 
from the ${ }^{5} \mathrm{D}_{0}$ level suggests that this line may be blended or incorrectly identified. However, not only is the ground-level $\lambda 1122.52$ line saturated, it is also contaminated by C I and possibly by vibrationally-excited $\mathrm{H}_{2}$. Since these effects tend to offset each other and the measurements of the excited levels are uncertain, we did not apply a multi-level calculation. For these reasons as well as the uncertain depletion of gas-phase Fe (see §3.3), our abundance estimate of Ge in NGC 3132 is less certain than for the other PNe.

\subsection{Correction for Dust Depletion}

The degree to which each element is depleted out of the gas and into the solid (dust) phase is an important issue for interpreting our results. As mentioned above, $\mathrm{S}$ is not significantly depleted in the ISM (Savage \& Sembach 1996). On the other hand, Fe is depleted by varying amounts in different components of the ISM: -1.25 dex in the warm ionized medium (WIM); as much as 2.27 dex in cold, neutral material (CNM; Savage et al. 1992). The Ge depletion has been measured along only a handful of sight lines; a value of -0.62 dex was determined by Cardelli et al. (1991), and Welty et al. (1999) found similar values, with slightly less depletion in WIM than in CNM gas.

Table 2 summarizes the Ge abundances we derive for each PN relative to the indicated reference ions. The two listed values correspond to the depletion patterns of the CNM and WIM, where we take the Fe depletions cited above. For Ge, we assume - 0.62 dex for the CNM case, and no depletion for the WIM, providing lower limits to the Ge abundances. It seems probable that conditions in PNe are most similar to those of the WIM, or intermediate, but the values in Table 2 cover the likely range of values.

\subsection{Ge Abundances and Implications}

The derived Ge abundances in these objects range from $\sim 3-30$ times solar, depending on the object and assumed depletion factors. We emphasize that the depletion of Ge is particularly uncertain, and that our values might be underestimates. Regardless, the qualitative result of this study is that Ge significantly enriched in the observed PNe.

It is interesting that the two best detections of Ge III, in SwSt 1 and $\mathrm{BD}+30^{\circ} 3639$, involve $\mathrm{PNe}$ with [WR] central stars. The surface compositions of these stars have presumably been altered by their prior evolution, since they exhibit severe $\mathrm{H}$ deficiencies and $\mathrm{C}$ enrichments. While the detailed evolutionary path which produces a [WR] central star is not well understood, it is likely to involve late thermal pulses, deep mixing, and/or heavy mass loss (Iben et al. 1993; Herwig 2001; Blöcker 2001). Therefore it is plausible that nucleosynthetic products such as s-process nuclei may be enhanced to a greater degree in nebulae with $[\mathrm{WR}]$ central stars than in other PNe. 


\section{SUMMARY}

We report the discovery of Ge III $\lambda 1088.46$ in four PNe observed with the Far Ultraviolet Spectroscopic Explorer. This line, which arises in the ionized zone, is used to estimate the abundance of $\mathrm{Ge}$ in the nebular gas relative to the elements $\mathrm{S}$ and $\mathrm{Fe}$, whose abundances are not altered by the star's evolution. We find convincing evidence for elevated abundances $\geq 3-10$ times solar (depending on assumed depletion factors) and therefore self-enrichment of Ge by $s$-process nucleosynthesis in the progenitor stars. This result demonstrates the potential of UV absorption-line spectroscopy to shed light on the operation of the $s$-process in PN progenitor stars. By determining the abundances of various heavy elements in $\mathrm{PNe}$, one can set constraints on models of stellar nucleosynthesis and mixing in late stellar evolutionary stages, and directly investigate the process of chemical enrichment of the ISM by stars that evolve through the AGB and PN phases.

We are grateful to the FUSE operations and science teams, whose exceptional efforts have made this facility available to the astronomical community. We thank S. McCandliss for providing data on $\mathrm{H}_{2}$, O. De Marco for sharing her STIS spectrum of SwSt 1, and D. Lambert and C.

Sneden for helpful comments. Financial support was provided by NASA contracts NAG5-9239, NAG5-11597, and NSF grant AST 97-31156.

\section{REFERENCES}

Abgrall, H., Roueff, E., Launay, F., Roncin, J. Y., \& Subtil, J. L. 1993a, A\&AS, 101, 273

Abgrall, H., Roueff, E., Launay, F., Roncin, J. Y., \& Subtil, J. L. 1993b, A\&AS, 101, 323

Blöcker, T. 2001, Ap\&SS, 275, 1

Bohigas, J., \& Olguín, L. 1996, RMxAA, 32, 47

Busso, M., Gallino, R., \& Wasserburg, G. J. 1999, ARA\&A, 37, 239

Busso, M., Gallino, R., Lambert, D.L., Travaglio, C., \& Smith, V.V. 2001, ApJ, 557, 802

Cardelli, J., Savage, B.D., \& Ebbets, D.C. 1991, ApJ, 383, L23

De Marco, O., Crowther, P. A., Barlow, M. J., Clayton, G. C., \& de Koter, A. 2001, MNRAS, 328, 527

De Marco, O. 2002, in Planetary Nebulae: IAU Symp. 209, in press

Dinerstein, H. L., Sneden, C., \& Uglum, J. 1995, ApJ, 447, 262

Dinerstein, H. L. 2001, ApJ, 550, L223

Grevesse, N., \& Noels, A. 1993, in Origin of the Elements, eds. N. Prantzos, E. Vangioni-Flam, M. Cassé (Cambridge: Cambridge Univ. Press), 15 
Herwig, F. 2001, Ap\&SS, 275, 15

Iben, I., Kaler, J. B., Truran, J. W., \& Renzini, A. 1983, ApJ, 264, 605

Kaler, J. B., \& Jacoby, G. 1991, ApJ, 372, 215

Käppeler, F. 1999, Prog. Particle \& Nuclear Phys., 43, 419

Käppeler, F., Beer, H., \& Wisshak, K. 1989, Rep. Prog. Phys., 52, 945

Méndez, R. H. 1978, MNRAS, 185, 647

Moos, H. W., et al. 2000, ApJ, 538, L1

Morton, D. C. 1991, ApJS, 77, 119

Morton, D. C. 2000, ApJS, 130, 403

Péquignot, D., \& Baluteau, J. P. 1994, A\&A, 283, 593

Pwa, T. H., Pottasch, S. R., \& Mo, J. E. 1986, A\&A, 164, 184

Savage, B. D., Cardelli, J. A., \& Sofia, U. J. 1992, ApJ, 401, 706

Savage, B. D., \& Sembach, K. R. 1996, ARA\&A, 34, 279

Sahnow, D. J., et al. 2000, ApJ, 538, L7

Sembach, K. R., Danks, A. C., \& Savage, B. D. 1993, A\&AS, 100, 107

Shaw, R. A., \& Dufour, R. J. 1995, PASP, 107, 896

Smith, V.V., \& Lambert, D.L. 1990, ApJS, 72, 387

Welty, D.E., Hobbs, L.M., Lauroesch, J.T., Morton, D.C., Spitzer, L., \& York, D.G. 1999, ApJS, 124,465 
Table 1. Line Fit Parameters

\begin{tabular}{|c|c|c|c|c|}
\hline Object & Ion & $\begin{array}{c}<v> \\
\left(\mathrm{km} \mathrm{s}^{-1}\right)\end{array}$ & $\begin{array}{c}W_{\lambda} \\
(\mathrm{m} \AA)\end{array}$ & $\begin{array}{c}N \\
\left(\mathrm{~cm}^{-2}\right)\end{array}$ \\
\hline \multirow[t]{7}{*}{ SwSt $1 \ldots .}$. & Ge III $\lambda 1088.46$ & $-42.2 \pm 0.5$ & $56 \pm 5$ & $(3.0 \pm 0.3) \times 10^{12}$ \\
\hline & Ge II $\lambda 1098.71$ & $\ldots$ & $<9.4$ & $<1.6 \times 10^{12}$ \\
\hline & Ge IV $\lambda 1189.03$ & $\ldots$ & $<90$ & $<1.4 \times 10^{13}$ \\
\hline & S III $\lambda 1012.50$ & $-42.3 \pm 1.3$ & $72 \pm 8$ & $(2.2 \pm 0.4) \times 10^{14}$ \\
\hline & S III* $\lambda 1015.78$ & $-38.6 \pm 0.4$ & $64 \pm 7$ & $(4.8 \pm 0.6) \times 10^{14}$ \\
\hline & S III** $\lambda 1021.33$ & $-47.6 \pm 0.9$ & $61 \pm 8$ & $(5.6 \pm 0.6) \times 10^{14}$ \\
\hline & S II $\lambda 1250.58^{\mathrm{a}}$ & $-39.2 \pm 2.9$ & $33 \pm 4$ & $(4.4 \pm 0.6) \times 10^{14}$ \\
\hline \multirow{6}{*}{$\mathrm{BD}+30^{\circ} 3639 \ldots$} & Ge III $\lambda 1088.46$ & $-82.3 \pm 0.6$ & $35 \pm 4$ & $(1.8 \pm 0.3) \times 10^{12}$ \\
\hline & Ge II $\lambda 1237.06$ & $\ldots$ & $<15$ & $<8.9 \times 10^{11}$ \\
\hline & Ge IV $\lambda 1229.84$ & .. & $<20$ & $<6.0 \times 10^{12}$ \\
\hline & S III $\lambda 1190.21$ & $-66.1 \pm 1.0$ & $99 \pm 7$ & $(2.7 \pm 0.2) \times 10^{14}$ \\
\hline & S III* $\lambda 1015.78$ & $-75.1 \pm 0.5$ & $47 \pm 3$ & $(2.4 \pm 0.4) \times 10^{14}$ \\
\hline & S III** $\lambda 1021.33$ & $-75.1 \pm 0.7$ & $53 \pm 12$ & $(2.1 \pm 0.6) \times 10^{14}$ \\
\hline \multirow[t]{5}{*}{ NGC $3132 \ldots}$. & Ge III $\lambda 1088.46$ & $-68.1 \pm 2.1$ & $30 \pm 6$ & $(1.6 \pm 0.3) \times 10^{12}$ \\
\hline & Ge II $\lambda 1098.71$ & $\ldots$ & $<20$ & $<4.4 \times 10^{12}$ \\
\hline & Fe III $\lambda 1122.52$ & $-52.6 \pm 1.0$ & $130 \pm 18$ & $(2.2 \pm 0.3) \times 10^{14}$ \\
\hline & Fe III* $\lambda 1124.88$ & $-52.8 \pm 2.0$ & $9.5 \pm 1.8$ & $(1.6 \pm 0.3) \times 10^{13}$ \\
\hline & Fe III* $\lambda 1130.40$ & $-49.8 \pm 0.7$ & $63 \pm 5$ & $(7.2 \pm 0.7) \times 10^{13}$ \\
\hline \multirow[t]{4}{*}{ IC $4593 \ldots .}$. & Ge III $\lambda 1088.46$ & $-2.9 \pm 2.0$ & $9.6 \pm 1.5$ & $(6.3 \pm 1.4) \times 10^{11}$ \\
\hline & Ge II $\lambda 1098.71$ & $\ldots$ & $<6.2$ & $<1.0 \times 10^{12}$ \\
\hline & S III* $\lambda 1015.78$ & $-5.5 \pm 0.5$ & $39 \pm 6$ & $(2.9 \pm 0.7) \times 10^{14}$ \\
\hline & S III** $\lambda 1021.33$ & $-5.5^{\mathrm{b}}$ & $20 \pm 8$ & $(8.1 \pm 4.1) \times 10^{13}$ \\
\hline
\end{tabular}

${ }^{a}$ Measured from STIS spectra.

${ }^{\mathrm{b}}$ Due to the weakness of S III** $\lambda 1021.33$, the central velocity was fixed to be the same as for S III* $\lambda 1015.78$. 
Table 2. Relative Germanium Abundances

\begin{tabular}{|c|c|c|c|c|c|}
\hline \multirow[b]{2}{*}{ Object } & \multirow{2}{*}{$\begin{array}{l}\text { Reference } \\
\text { Ion }\end{array}$} & \multicolumn{2}{|c|}{$N($ Ge III $) / N\left(X^{+i}\right)$} & \multicolumn{2}{|c|}{$[\mathrm{Ge} / \mathrm{X}]$} \\
\hline & & $\mathrm{CNM}^{\mathrm{a}}$ & WIM $^{b}$ & $\mathrm{CNM}^{\mathrm{a}}$ & WIM $^{b}$ \\
\hline SwSt 1 & S II, S III & $(6.9 \pm 2.2) \times 10^{-3}$ & $(1.6 \pm 0.5) \times 10^{-3}$ & $1.65 \pm 0.15$ & $0.86 \pm 0.15$ \\
\hline $\mathrm{BD}+30^{\circ} 3639$ & S II, S III & $(3.6 \pm 1.3) \times 10^{-3}$ & $(8.6 \pm 3.1) \times 10^{-4}$ & $1.18 \pm 0.17$ & $0.57 \pm 0.17$ \\
\hline NGC 3132 & Fe III & $(1.6 \pm 0.4) \times 10^{-4}$ & $(4.1 \pm 1.0) \times 10^{-4}$ & $0.09 \pm 0.12$ & $0.49 \pm 0.12$ \\
\hline IC 4593 & $\mathrm{~S}$ II, S III ${ }^{\mathrm{d}}$ & $(3.6 \pm 1.5) \times 10^{-3}$ & $(8.5 \pm 3.5) \times 10^{-4}$ & $1.20 \pm 0.19$ & $0.57 \pm 0.19$ \\
\hline
\end{tabular}

Note. - Columns (3) and (4) give column density ratios $N($ Ge III $) / N\left(\mathrm{X}^{+i}\right)$ where $\mathrm{X}$ is the indicated reference ion ( $\mathrm{S}$ or Fe); values in square brackets in Columns (5) and (6) are in logarithmic units with respect to the meteoritic values of Grevesse \& Noels (1993). Cited errors are propagated from the values in Table 1 and corrections made for depletion and unobserved levels using Shaw \& Dufour's (1995) web-based 5-level program.

${ }^{a}$ Column densities of Ge III and Fe III adjusted for depletions in the cold interstellar clouds toward $\zeta$ Oph (Savage et al. 1992; Savage \& Sembach 1996). Sulfur is assumed to be undepleted.

${ }^{\mathrm{b}}$ Column density of Fe III adjusted for depletion seen in the warm diffuse cloud toward $\zeta$ Oph (Savage et al. 1992; Savage \& Sembach 1996). Ge and S are assumed to be undepleted.

${ }^{c}$ Assumes $\left(\mathrm{S}^{+} / \mathrm{S}^{++}\right)$from Pwa et al. (1986).

${ }^{\mathrm{d}}$ Assumes $\left(\mathrm{S}^{+} / \mathrm{S}^{++}\right)$from Bohigas \& Olguín (1996). 
Fig. 1.- Continuum normalized absorption spectra are plotted against heliocentric velocity for the indicated species. Profiles for each object are arranged by column, with reference lines below Ge III $\lambda 1088.46$. The S III $\lambda 1190.21$ profile is from STIS data, while all other features shown were obtained by FUSE. Note that the vertical scale is enlarged for IC 4593, which has the weakest Ge III line. Other ionic lines are indicated by species name and $\mathrm{H}_{2}$ lines by vertical tick marks. The heavy solid lines are modeled Gaussian fits to nebular absorptions; other lines modeled in the fitting process are shown as dashed lines. The vertical dotted lines indicate the nebular velocities measured from Na I and other species. We were unable to fit S III $\lambda 1012.50$ in IC 4593 due to blending with $\mathrm{H}_{2}$. 
Normalized Intensity
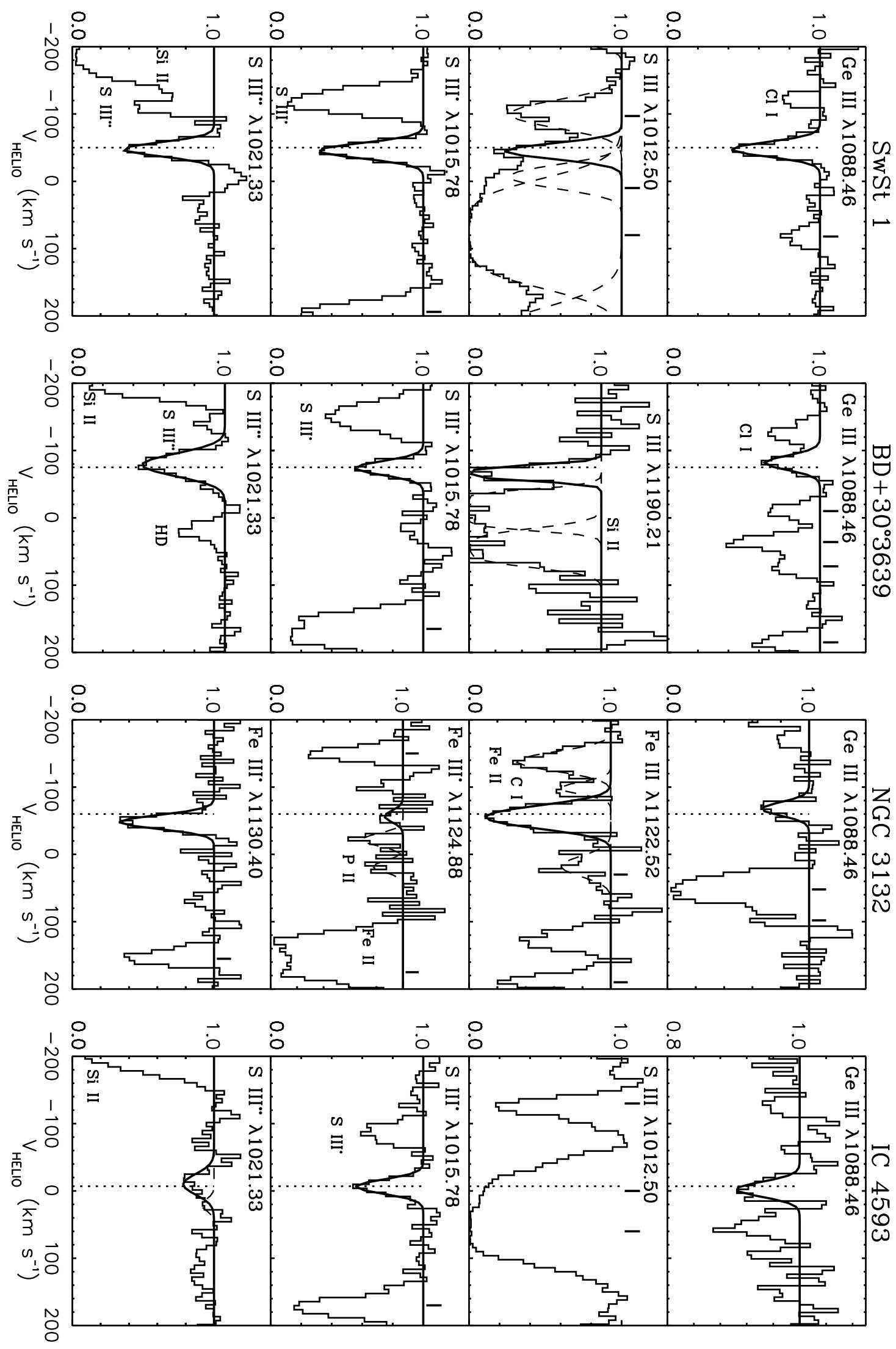